\title{
Validez de constructo y confiabilidad de la Escala de Satisfacción con la Vida, en escolares de zonas rurales y urbanas de Cundinamarca
}

\section{Construct validity and reliability of the Satisfaction with Life Scale, in schoolchildren from rural and urban areas of Cundinamarca}

\author{
Laura Vanessa Hernández Mateus ${ }^{1}$ \\ Sergio Andrés Salamanca Chacón ${ }^{2}$ \\ Lina Isabel Umbarila Contreras ${ }^{3}$ \\ Carlos Francisco Venegas Muñoz ${ }^{4}$ \\ Fernando Riveros Munévar ${ }^{5}$
}

Universidad de San Buenaventura, Colombia

\begin{abstract}
The aim of the present instrumental study was to determine the validity of the construct and reliability of the Satisfaction with Life Scale, in schoolchildren from rural and urban areas of Cundinamarca. The study involved the application of the scale on 258 schoolchildren from the city of Bogotá and the municipalities of Subachoque and Cogua, Cundinamarca, aged between 12 and 18 years of age $(M=15.03, S D=1.219)$, carrying out exploratory and confirmatory factor analyses, as well as an analysis of reliability and internal consistency. The results show a unifactorial scale, which explains the $57.669 \%$ of the total variance, with said unifactorial model confirmed, a Cronbach's alpha of .814 , positive correlations among all the reagents and between the items with the total of the scale, which allows us to conclude/state that the Satisfaction with Life Scale has adequate validity and reliability indexes to be used as a measure in schoolchildren in rural and urban areas of Cundinamarca.

Keywords. Construct validity, reliability, life satisfaction.

Resumen. El objetivo del presente estudio de tipo instrumental fue determinar la validez de constructo y confiabilidad de la Escala de Satisfacción con la Vida, en escolares de zonas rurales y urbanas de Cundinamarca. Para ello, se contó con la participación de 258 escolares de la ciudad de Bogotá y de los municipios de Subachoque y Cógua, Cundinamarca, con edades comprendidas entre los 12 y los 18 años $(M=15.03$, DT $=1.219)$; se les aplicó la escala, llevando a cabo análisis factoriales exploratorios y confirmatorios, así como análisis de confiabilidad y consistencia interna. Los resultados muestran una escala unifactorial, que explica el 57.669\% de la varianza total, con dicho modelo unifactorial confirmado, un alfa de Cronbach de .814, correlaciones positivas entre todos los reactivos y entre los ítems con el total de la escala, lo que permite afirmar que la Escala de Satisfacción con la Vida cuenta con adecuados índices de validez y confiabilidad para ser utilizada como una medida en escolares de zonas rurales y urbanas de Cundinamarca.
\end{abstract}

Palabras clave. Validez de constructo, confiabilidad, satisfacción con la vida.

${ }^{1}$ Laura Vanessa Hernández Mateus. Universidad de San Buenaventura, Colombia. Dirección Postal: 110141. E-mail: Lvanesaa2603@gmail.com.

${ }^{2}$ Sergio Andrés Salamanca Chacón. Universidad de San Buenaventura,Colombia. E-mail: shogun.sergio@gmail.com ${ }^{3}$ Lina Isabel Umbarila Contreras. Universidad de San Buenaventura, Colombia. E-mail: linaisabel_96@hotmail.com ${ }^{4}$ Carlos Francisco Venegas Muñoz. Universidad de San Buenaventura,Colombia. E-mail: francisco.994@hotmail.com ${ }^{5}$ Fernando Riveros Munévar. Universidad de San Buenaventura, Colombia. E-mail: efriveros45@hotmail.com

\section{@ $\odot \Theta \Theta$}

Esta obra está bajo una licencia de Creative Commons Reconocimiento-NoComercial-SinObraDerivada 4.0 Internacional. 


\section{Introducción}

La satisfacción con la vida es un factor fundamental en lo que respecta al constructo de bienestar subjetivo de los seres humanos (Castaños \& Sánchez, 2015). Al estudiarlo, se ha encontrado que es un componente cognitivo en el que se hacen apreciaciones valorativas sobre aspectos globales de la calidad de vida que se lleva (Marrero \& Carballeira, 2010).

En concreto, la satisfacción con la vida es el grado en el que una persona evalúa su calidad global de vida (Pavot \& Diener, 1993; Veenhoven, 1994; Diener, 2000). En dicha evaluación, el individuo examina los aspectos tangibles, valora lo bueno contra lo malo, lo compara con un estándar o criterio elegido y llega a un juicio sobre la satisfacción con su vida (Castaños \& Sánchez, 2015).

Es importante resaltar que, en este proceso, la persona evalúa tanto los diferentes ámbitos y condiciones, los objetivos planeados y conseguidos, así como los hechos pasados (Infante \& Goñi, 2010). De igual modo, es relevante mencionar que, aunque la satisfacción tiende a ser estable, se encuentra susceptible a las circunstancias cambiantes del medio, bien sea a nivel familiar, económico, académico, social y/o de salud tanto física como mental (Ramírez \& Lee, 2012).

Teniendo en cuenta lo anterior, cabe mencionar que la satisfacción con la vida depende en gran medida del balance personal que manifiesta cada individuo, ya que este debe llenar de contenido y de nuevas metas la existencia de quien se evalúa (Aranda \& Vara, 2006). En un intento por mejorar la satisfacción con la vida, se debe reducir el afecto negativo y aumentar el positivo, puesto que se asume que la ausencia de afecto negativo no es lo mismo que la presencia del positivo; es decir, mientras más frecuente se sienta el uno, menos frecuente se percibe el otro (Gómez, Villegas, Barrera, \& Cruz, 2007).

Con respecto a cómo medir este constructo, la Escala de Satisfacción con la Vida es un instrumento simple, sencillo, de fácil y de amplio uso, conformado por cinco reactivos que se estructuran en un único factor. Varias investigaciones a nivel mundial resaltan las propiedades métricas de dicha escala, refiriendo la unidimensionalidad y la alta consistencia interna de la misma así como su utilidad y practicidad. Al respecto, uno de los primeros estudios de este estilo (Diener, Emmons, Larsen, \& Griffin, 1985), el cual refirió precisamente un único factor que explica el $66 \%$ de la varianza acumulada. El estudio fue replicado por Pavot y Diener (1993), quienes hallaron la misma estructura factorial, ambos con muestras no clínicas. En investigaciones más actuales, con mejores métodos de análisis y llevados a cabo en poblaciones de diversos rangos etarios, condiciones sociales y lenguas, también refieren un único factor con adecuada consistencia interna (Diener, Tay, \& Oishi, 2013; Kowalska, Gorackzo, Javorwka, \& Szczepańska, 2017; Ćwiek et al., 2017).

Estudios sobre su validez y confiabilidad en población hispanohablante no clínica han sido realizados recientemente en España (Vázquez, Duque, \& Hervás, 2013) y otros más en América Latina (Padros, Gutiérrez, \& Medina, 2015; Vera et al., 2012; Moyano, Martínez, \& Muñoz, 2013), los cuales hallaron, también, la unidimensionalidad y las adecuadas condiciones de consistencia interna (alfas superiores a .85). Sin embargo, no se cuenta con datos para Colombia, menos aún población escolarizada, por lo 
que el objetivo de esta investigación fue determinar la validez de constructo y la confiabilidad de la Escala de Satisfacción con la Vida (SWLS) para escolares de zonas rurales y urbanas de Cundinamarca.

\section{Método}

Tipo de estudio

La presente investigación se enmarca como un estudio de corte transversal, de alcance descriptivo y de tipo instrumental (Montero \& León, 2005).

\section{Participantes}

Se contó con la participación de 258 escolares de la ciudad de Bogotá y de los municipios de Subachoque y Cógua, Cundinamarca; sus edades estaban comprendidas entre los 12 y los 18 años $(M=15.03, D T=1.219)$ y fueron distribuidos en 194 estudiantes de colegios privados y 64 de colegios públicos, tanto rurales como urbanos; de ellos, 134 eran mujeres y 124 hombres, los cuales se hallaban entre los cursos octavo a once de formación básica secundaria. Como criterios de inclusión, los participantes debían cumplir con el consentimiento y asentimiento informado, estar en los rangos de edad, no tener enfermedades de alto impacto al momento de la aplicación, presentar regularidad cognitiva (garantizado este criterio por la institución) y no tener diagnósticos clínicos psicológicos o psiquiátricos (garantizado con preguntas a cada participante). El tipo de muestro fue no probabilístico por sujetos disponibles.

Cabe referir que este tamaño muestral se obtuvo garantizando la regla de Kaiser de 10 aplicaciones por reactivo (para este estudio se tuvieron 50 aplicaciones por reactivo) y la pertinencia muestral y de matriz de correlaciones con las pruebas de Kaiser-Meyer-Olkin y de esfericidad de Bartlett.

\section{Instrumentos}

Se utilizó la Escala de Satisfacción con la Vida (SWLS) de Diener et al. (1985). Este es un instrumento de 5 ítems en escala tipo Likert de 7 puntos que van desde 1 como "muy en desacuerdo" y 7 como "muy de acuerdo"; las puntuaciones van de 5 a 35 y que evalúa la satisfacción general que tiene el individuo con su vida, entendiendo que mayor puntuación refleja mayor satisfacción. En este estudio se contó con la versión traducida y adaptada al español por Vásquez, Duque y Hervás (2013).

\section{Procedimientos}

Posterior a la firma del consentimiento informado en los padres y de los asentimientos en los escolares, se procedió a realizar las aplicaciones del inventario, las cuales se realizaron en los respectivos salones de clase, garantizando la misma instrucción a todos los niños y en espacios que no permitiera el diálogo entre ellos. Los datos fueron analizados a través del paquete estadístico SPSS, versión 24. Inicialmente se realizó la eliminación de datos atípicos con la prueba de distancias de extremas de Mahalanobis, seguido del análisis del tamaño muestral con la prueba de adecuación de Kaiser-Meyer-Olkin (se esperaba obtener un valor superior a.7). También, se efectuó un análisis de la adecuación de la matriz de correlaciones con la prueba de esfericidad de Barlett (esperando una significancia inferior 
al .05). Posterior a ello se llevó a cabo un análisis factorial exploratorio con un método de extracción de máxima verosimilitud y una rotación oblicua; un análisis de confiabilidad y consistencia interna con alfa de Cronbach general y con simulación de eliminación de cada reactivo; correlaciones entre los reactivos y correlaciones entre cada ítem y el total corregido. Se usó el aplicativo Amos para realizar el análisis factorial confirmatorio con un método de extracción de máxima verosimilitud, teniendo en cuenta una matriz de tipo policórica, y se analizó la división entre chi cuadrado $(p<.05)$ y los grados de libertad $\left(\chi^{2} / g \emptyset\right)$, que deben ser menor a tres. Por último, se realizó el índice de bondad de ajuste (GFI), el cual también se estandarizó (AGFI) y el índice de ajuste comparativo (CFI), de estos tres se esparaban coeficientes superiores a .95) y el error cuadrático medio de aproximación (RMSEA) inferior a .05. Es importante indicar que se garantizó la normalidad multivariada al verificar los índices de asimetría y curtosis, en un umbral entre -1.5 y +1.5 , y que la base de datos total se utilizó para el análisis exploratorio y una muestra aleatorizada de la misma para el análisis confirmatorio.

\section{Resultados}

Con el fin de establecer si el tamaño muestral es apropiado para realizar los análisis factoriales, se procedió a realizar la prueba Kaiser-Meyer-Olkin de adecuación de muestreo y la prueba de esfericidad de Bartlett, encontrando un KMO de .831 y una significancia en Bartlett de .001, lo que permite indicar que el tamaño muestral es pertinente y que la matriz de covariaciones soporta el análisis factorial. Se procedió, entonces, a realizarse dicho análisis con el método de extracción de componentes principales y rotación varimax. En la tabla 1 se muestran las extracciones obtenidas para cada reactivo.

Los datos obtenidos en la tabla 1 refieren que la totalidad de los ítems muestran una extracción superior a .4, lo que refiere que es apropiado conservar todos los reactivos. Con respecto al número de factores, se encontró un único factor que explica el 57.669\% de la varianza total acumulada y donde todos los ítems arrojan una carga entre 0.664 y 0.860 . Dada la estructura unifactorial obtenida, se llevó a cabo el análisis factorial confirmatorio con una muestra aleatoria de la aplicación general $(n=100)$, por medio del aplicativo Amos, con la que se encontró el modelo gráfico confirmado en la figura 1.

Tabla 1

Extracciones de los reactivos del SWLS

\begin{tabular}{ccc}
\hline Reactivo & Inicial & Extracción \\
\hline Ítem 1 ESV & 1 & .522 \\
Ítem 2 ESV & 1 & .567 \\
Ítem 3 ESV & 1 & .739 \\
Ítem 4 ESV & 1 & .614 \\
Ítem 5 ESV & 1 & .441 \\
\hline
\end{tabular}

Actualidades en Psicología, 32(125), 2018, 71-78 


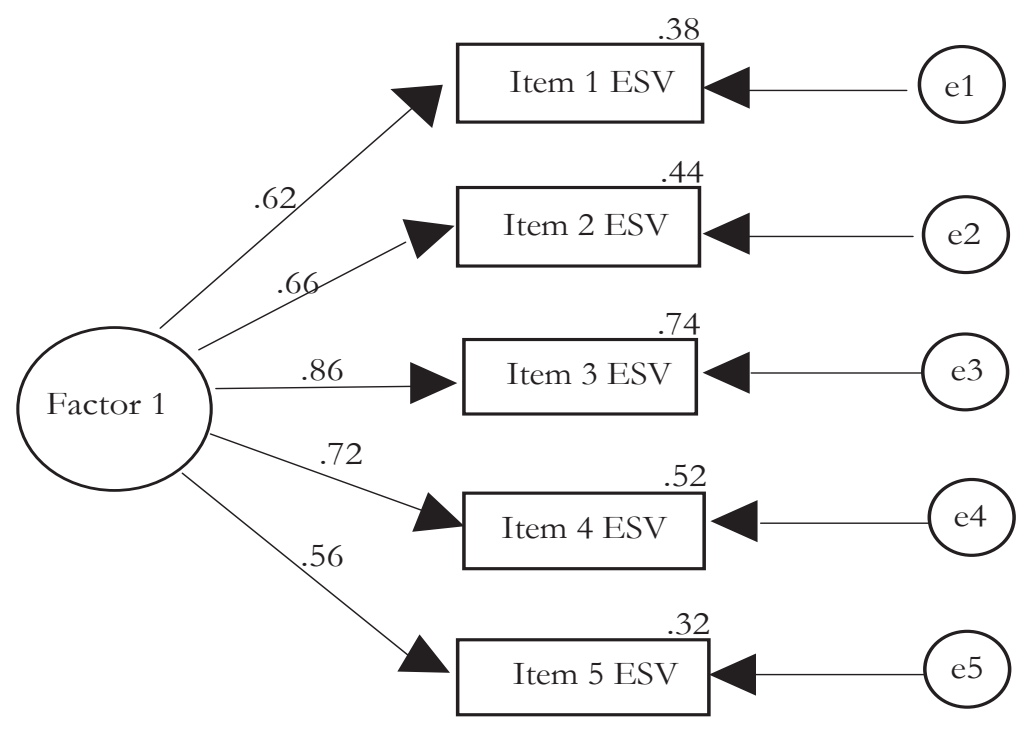

Figura 1. Modelo factorial confirmado del SWLS, con cargas estandarizadas

Tabla 2

Matriz de correlaciones entre los reactivos del SWLS

\begin{tabular}{lccccc}
\hline & 1 & 2 & 3 & 4 & 5 \\
\hline Item 1 ESV & 1 & .451 & .520 & .442 & .344 \\
Item 2 ESV & & 1 & .559 & .475 & .359 \\
Item 3 ESV & & & 1 & .628 & .497 \\
Item 4 ESV & & & & 1 & .389 \\
Item 5 ESV & & & & & 1 \\
\hline
\end{tabular}

Los resultados encontrados en la figura 1 muestran cargas altas y positivas entre los reactivos y el factor, y estadísticos apropiados obtenidos en el factorial confirmatorio $\left(\chi^{2}=2.394\right.$; $\left.g l=5 ; \chi^{2} / g l=.479 ; \mathrm{GFI}=.996 ; \mathrm{AGFI}=.989 ; \mathrm{CFI}=1 ; \mathrm{RMSEA}=.001\right)$, que garantizan que el modelo unifactorial tiene soporte suficiente. Ahora, con respecto a confiabilidad, se encontró un alfa de Cronbach de .814. La tabla 2 muestra las correlaciones positivas y superiores a .3 entre todos los ítems de la escala, mientras que la tabla 3 refiere altas correlaciones entre cada ítem con el total corregido de la escala y afectaciones al alfa en caso hipotético de eliminar un reactivo, lo que habla de la consistencia interna de la escala. 
Tabla 3

Correlaciones entre elemento-total corregida y alfas tras la supresión de cada item

\begin{tabular}{lcc}
\hline & $\begin{array}{c}\text { Correlación total } \\
\text { de elementos corregida }\end{array}$ & $\begin{array}{c}\text { Alfa de Cronbach si el } \\
\text { elemento se ha suprimido }\end{array}$ \\
\hline Item 1 ESV & .556 & .782 \\
Item 2 ESV & .589 & .772 \\
Item 3 ESV & .735 & .726 \\
Item 4 ESV & .625 & .761 \\
Item 5 ESV & .500 & .809 \\
\hline
\end{tabular}

\section{Discusión}

El estudio de las condiciones no patológicas de los seres humanos ha tomado alta importancia. Precisamente la satisfacción con la vida se ha tornado un tema de alto impacto a nivel internacional y, si bien existen instrumentos objetivos tales como la Escala de satisfacción con la vida, se desconocen sus propiedades psicométricas para población colombiana, lo que motivó la presente investigación.

En torno a los hallazgos principales, se encontró que la Escala de satisfacción con la vida (SWLS) es un instrumento conformado por un único factor, que explica el 57.669\% de la varianza total acumulada, con una carga de cada ítem a dicho componente entre .664 y .860. Adicional, este modelo fue sometido a un análisis factorial confirmatorio, donde se rectifica la coherencia entre el modelo teórico y el modelo empírico obtenido, lo que rectifica la apropiada validez de constructo para la muestra trabajada. Finalmente, y en lo que respecta a confiabilidad, se halló un alfa de Cronbach de .814, lo que junto a las correlaciones positivas y superiores a .3 entre todos los ítems de la escala, a las altas correlaciones entre cada ítem con el total corregido de la escala y las afectaciones al alfa en caso hipotético de eliminar un reactivo, permiten indicar que la escala SWLS es un instrumento confiable y consistente para la muestra de la investigación.

Los hallazgos anteriores son similares a otros estudios previos: los apropiados índices de validez así como la unifactorialidad hallada concuerdan con los datos de Diener et al. (1985), la revisión de Pavot y Diener (1993) y el estudio de Diener, Tay y Oishi (2013). Además, este último encontró un alto alfa de Cronbach para la escala. De igual forma, las apropiadas correlaciones entre los reactivos también fueron halladas por Pavot y Diener (1993). También, debe indicarse que los hallazgos encontrados son equivalentes a los realizados en España y en algunos países latinoamericanos (Vera et al., 2012; Moyano, Martínez, \& Muñoz, 2013; Padros, Gutiérrez, \& Medina, 2015); sin embargo, ninguno de los estudios previamente nombrados llevó a cabo el análisis factorial confirmatorio, por lo que el presente estudio es pionero en torno al uso de este análisis de ecuaciones estructurales para la confirmación de un modelo factorial en dicho instrumento. 
Por otro lado, deben nombrarse algunas limitantes encontradas en el presente estudio. Primero, si bien los estadísticos de adecuación muestral indicaron que el número de participantes era suficiente para llevar a cabo los demás análisis estadísticos, se considera que el tamaño igualmente puede ser reducido para considerarse representativo de los escolares de zonas urbanas y rurales de Cundinamarca, por lo que es necesario replicar los hallazgos en otras muestras similares. Adicionalmente, los participantes pertenecen a dos municipios y a la ciudad, por lo que se recomienda que, también, se lleve a cabo este estudio con muestras de otros municipios de Cundinamarca, lo que permitiría rectificar los hallazgos obtenidos. Otra limitación del presente estudio es el tipo de muestreo no probabilístico, por lo que se recomienda replicar esta investigación con muestras aleatorias.

En conclusión, se puede indicar que la Escala de Satisfacción con la Vida (SWLS) es un instrumento con apropiados índices de validez, consistencia y confiabilidad para escolares de zonas rurales y urbanas de Cundinamarca, por lo que puede utilizarse para medir dicho constructo en estas poblaciones. De igual forma, se recomiendan futuras investigaciones para replicar estos hallazgos en otros lugares de Colombia, verificando principios de invarianza factorial por sexo, edades, condiciones socioeconómicas, lugares del país entre otras variables; además, con otros grupos poblacionales (jóvenes, adultos mayores, enfermos crónicos, entre otros) y futuros estudios donde se evidencie la correlación con otros instrumentos similares como evidencias adicionales de la validez de constructo y como referencia a la validez de criterio. Adicionalmente, se proponen futuros estudios para determinar precisamente los niveles de satisfacción con la vida en escolares de distintos contextos y condiciones en el país, realizar otras investigaciones dirigidas a establecer otras variables que pueden afectar o relacionarse con esta y, finalmente, a establecer los efectos de programas de intervención para mejorar la satisfacción con la vida en escolares colombianos.

\section{Referencias}

Aranda, J., \& Vara, A. (2006). Factores asociados a la satisfacción de vida de adultos mayores de 60 años en Lima-Perú. Universitas Psychologica, 5(3), 475-485. Recuperado de http:/ / www.redalyc.org/articulo.oa?id=64750304

Castaños, S., \& Sánchez, J. (2015). Niñas y adolescentes en riesgo de calle: Bienestar subjetivo y salud mental. CES psicología, 8(1), 120-133.

Ćwiek, A., Czok, M., Kurczab, B., Kramarczyk, K., Drzyzga, K., \& Kucia, K. (2017). Association between depression and hemodialysis in patient with chronic kidney disease. Psychiatria Danubina, 29(3), 499-503. Recuperado de http://www.hdbp. org/psychiatria_danubina/pdf/dnb_vol29_sup3/dnb_vol29_sup3_499.pdf

Diener, E. (2000). Subjective well-being: The science of happiness and a proposal for a national index. American Psychologist, 55(1), 34 -43. doi: 10.1037/0003-066X.55.1.34

Diener E., Emmons R., Larsen R. J., \& Griffin S. (1985). The Satisfaction with Life Scale. Journal of Personality Assessment, 49(1), 71-75. doi: 10.1207/s15327752jpa4901_13 
Diener, E., Tay, L., \& Oishi, S. (2013). Rising income and subjective Well-Being of Nations. Journal of Personality and social Psychology, 104(2), 267-276.

Gómez, V., Villegas, C., Barrera, F., \& Cruz, J. (2007). Factores predictores de bienestar subjetivo en una muestra colombiana. Revista Latinoamericana de Psicología, 39(2), 311-325. Recuperado de http://www.redalyc.org/ articulo.oa?id=80539208

Infante, G. \& Goñi, E. (2010). Actividad físico-deportiva, autoconcepto físico y satisfacción con la vida. European Journal of Education and Psychology, 3(2), 199-208. doi: 10.1989 / ejep.v3i2.60

Kowalska, J., Gorackzo, A., Javorwka, L., \& Szczepańska, J. (2017). An Assessment of the Burden on Polish Caregivers of Patients With Dementia: A Preliminary Study. American Journal of Alzheimer's Disease and Other Dementias, 32(8), 509-515. doi: $10.1177 / 1533317517734350$

Marrero, R., \& Carballeira, M. (2010). El papel del optimismo y del apoyo social en el bienestar subjetivo. Salud Mental, 33(1), 39-46. Recuperado de: http://www.scielo.org.mx/ scielo.php?script $=$ sci_arttext\&pid=S0185-33252010000100005\& lng=es\&tlng=es

Montero, I. \& León, O. (2005). Sistema de clasificación del método en los informes de investigación en Psicología. International Journal of Clinical and Health Psychology, 5(1), 115-127.

Moyano, N., Martínez, M., \& Muñoz, M. (2013). Propiedades psicométricas de la escala de satisfacción con la vida de Diener. Revista Argentina de Clínica Psicológica, 22(2), 161-168.

Ramírez, M. \& Lee, S. (2012). Factores asociados a la satisfacción vital en adultos mayores de 60 años. Revista Latinoamericana Polis, 33, 1-15. Recuperado de: https://polis.revues.org/8594

Padrós, F., Gutiérrez, C., \& Medina, M. (2015). Propiedades psicométricas de la escala de satisfacción con la vida (SWLS) de Diener en población de Michoacán (México). Avances en Psicología Latinoamericana, 33(2), 223-232. doi: 10.12804/apl33.02.2015.04

Pavot, W. \& Diener, E. (1993). The affective and cognitive contest of self-reports measures of subjective well-being. Social Indicators Research, 28(1), 1-20. doi: 10.1007/BF01086714

Vásquez, C., Duque, A., \& Hervás, G. (2013). Satisfaction with Life Scale in a Representative Sample of Spanish Adults: Validation and Normative Data. Spanish Journal of Psychology, 16, e82, 1-15. doi: 10.1017/sjp.2013.82

Veenhoven, R. (1994). El estudio de la satisfacción con la vida. Intervención Psicosocial, 3, 87-116. Recuperado de http://h dl.handle.net/1765/16195

Vera-Villarroel, P., Urzúa, M. A., Pavez, P., Celis-Atenas, K., \& Silva, J. (2012). Evaluation of Subjective Well-being: Analysis of the Satisfaction with Life Scale in Chilean Population. Universitas Psychologica, 11(3), 719-727.

Recibido:17 de julio de 2017

Aceptado: 12 de julio de 2018 